\title{
ON THE CHARACTERISTIC CLASSES OF ACTIONS OF LATTICES IN HIGHER RANK LIE GROUPS
}

\author{
GARRETT STUCK
}

\begin{abstract}
We show that under certain assumptions, the measurable cohomology class of the linear holonomy cocycle of a foliation yields information about the characteristic classes of the foliation. Combined with the results of a previous paper, this yields vanishing theorems for characteristic classes of certain actions of lattices in higher rank semisimple Lie groups.
\end{abstract}

Let $\Gamma$ be a discrete group acting by diffeomorphisms on a smooth compact manifold $M$. Associated to this action are certain characteristic classes in $H \cdot(\Gamma, \mathbf{R})$, which are constructed as characteristic classes of a natural foliation associated to the group action. The action of $\Gamma$ on $M$ induces an action of $\Gamma$ on the principal frame bundle $P(M)$ of $M$ and the characteristic classes of the action can be interpreted as obstructions to the existence of invariant geometric structures on $M$, i.e., principal subbundles of $P(M)$ invariant by the $\Gamma$-action.

If $\Gamma$ is a lattice in a higher rank semisimple Lie group, then $\Gamma$ has strong rigidity properties (see, e.g., [M, Z1]). In an earlier paper [S], we showed, using techniques from ergodic theory, that for a certain class of $\Gamma$-actions there is always an invariant measurable reductive geometric structure, i.e., a measurable principal subbundle with reductive structure group, which is invariant by the $\Gamma$-action. Moreover, the noncompact semisimple part of this reductive group is locally isomorphic to a semisimple factor of the ambient Lie group of $\Gamma[Z 1]$. Zimmer [Z3] recently proved this result for a large class of actions (which does not a priori include the class of actions considered in [S]). A natural question is whether these results remain true in the smooth category. The purpose of this paper is to show that the characteristic classes, which obstruct a smooth geometric structure, vanish in the presence of a measurable geometric structure. Explicitly, we have

Main Theorem. Let $(M, \mathscr{F})$ be a codimension $n, C^{2}$-foliated manifold and suppose that the linear holonomy cocycle is measurably equivalent to a locally tempered cocycle taking values in a subgroup $H \subset G L(n, \mathbf{R})$ which is stable under transpose. Then the Weil homomorphism

$$
\chi: H^{\cdot}(\mathfrak{g l}(n), O(n)) \rightarrow H_{c}^{\cdot}(M, \mathscr{F})^{*}
$$

Received by the editors February 24, 1989.

1980 Mathematics Subject Classification (1985 Revision). Primary 57R32; Secondary 57S20.

Research partially supported by an Alfred P. Sloan Dissertation Fellowship. 
factors through $H^{\cdot}(\mathfrak{h}, H \cap O(n))$. In particular, if the restriction of $\tau \in$ $H^{k}(\mathfrak{g l}(n), O(n))$ to $H^{k}(\mathfrak{h}, H \cap O(n))$ vanishes, then $\chi(\tau): H_{c}^{m-k}(M, \mathscr{F}) \rightarrow \mathbf{R}$ is zero.

The theorem follows immediately from the functoriality of the characteristic homomorphism in the case that the linear holonomy cocycle is smoothly equivalent to a cycycle of the specified form. We note in passing that the Main Theorem holds for measurable, leaf saturated subsets of $M$, i.e., for the Weil measures $[\mathrm{H}-\mathrm{H}]$ associated to $\mathscr{F}$. We leave it to the reader conversant with these notions to formulate and deduce the more general statement.

Combined with the results of [S and Z3], the previous theorem yields the

Main Corollary. Let $G$ be a semisimple Lie group with finite center, all of whose simple factors have real rank at least two. Let $\Gamma$ be a lattice in $G$ and suppose $\Gamma$ acts on a smooth manifold $M$ preserving a finite measure. Assume that either

(i) $M$ is compact and $\Gamma$ is cocompact, or

(ii) $\Gamma$ is irreducible, the action of $\Gamma$ on $M$ is mixing, and dimension of $M$ is smaller than the minimum dimension of an almost faithful real representation of $G$.

Then there is a reductive subgroup $H \subset G L(n, \mathbf{R})$ with compact center such that the Weil homomorphism of the associated foliation of $G / K \times_{\Gamma} M$ factors through $H^{\cdot}(\mathfrak{h}, M)$, where $M$ is the maximal compact subgroup of $H$. Moreover, the noncompact semisimple part of $H$ is locally isomorphic to a semisimple factor of $G$.

It is straightforward to show, using the Margulis super-rigidity theorem, that if $M$ is a homogeneous manifold $L / \Lambda$ and the action of $\Gamma$ on $M$ is given by a homomorphism of $\Gamma$ into $L$, then there is a smooth $\Gamma$-invariant reduction of $P(M)$ to a reductive group. The Main Corollary gives evidence that every volume preserving action of $\Gamma$ enjoys this property of homogeneous actions.

Hurder and Katok [H-K] proved a version of the Main Theorem for amenable foliations; they showed (roughly speaking) that the linear holonomy cocycle of an amenable foliation is measurably equivalent to a tempered cocycle into a maximal amenable subgroup of $G L(n, \mathbf{R})$ and then that the Weil homomorphism factors through the relative Lie algebra cohomology of the Lie algebra of the amenable group. They also proved a slightly weaker version of the Main Theorem for $H=S L(n, \mathbf{R})$, but their proof does not extend to the general case.

The structure of the paper is as follows: In the first section we define characteristic classes of foliations and the Weil homomorphism. At the end of $\S 1$ we give a brief overview of the proof of the Main Theorem. In $\S 2$ we define the measurable equivalence class of the linear holonomy cocycle and in $\S 3$ we discuss a procedure for averaging in noncompact symmetric spaces. $\S \S 4,5$, and 6 complete the proof of the Main Theorem and in $\S 7$ we discuss the Main Corollary. 


\section{Characteristic Classes of foliations}

Characteristic classes of foliations have been constructed in various ways by several authors. We follow the presentation given in [K-T].

Let $\mathfrak{g}$ be a (real) Lie algebra and $\mathfrak{g}^{*}$ its dual. Define the Weil algebra of $\mathfrak{g}$ to be $W(\mathfrak{g})=\bigwedge \mathfrak{g}^{*} \otimes S \mathfrak{g}^{*} . W(\mathfrak{g})$ has a bigrading $W^{q, 2 p}(\mathfrak{g})=\bigwedge^{q} \mathfrak{g}^{*} \otimes S^{p} \mathfrak{g}^{*}$ and with the associated total grading $W(\mathfrak{g})$ is a graded commutative algebra. For $X \in \mathfrak{g}$ let $i(X)$ be the unique derivation of degree -1 on $W(\mathfrak{g})$ such that $i(X) \alpha=\alpha(X)$ for $\alpha \in \Lambda^{1} \mathfrak{g}^{*}$ and $i(X) \beta=0$ for $\beta \in S^{1} \mathfrak{g}^{*}$. Let $\theta(X)$ be the unique derivation of degree 0 on $W(\mathfrak{g})$ such that $\theta(X)=(\operatorname{ad} X)^{*}$ on $\Lambda^{1} \mathfrak{g}^{*}$ and $S^{1} \mathfrak{g}^{*}$. There is a differential of degree 1 on $W(\mathfrak{g})$ defined as follows: for

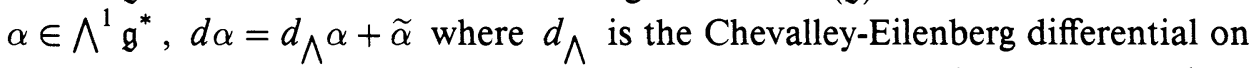
$\Lambda \mathfrak{g}^{*}$ and $\widetilde{\alpha}$ is the 1 -form $\alpha$ considered as an element of $S^{1} \mathfrak{g}^{*}$; for $\beta \in S^{1} \mathfrak{g}^{*}$, $d \beta \in \Lambda^{1} \mathfrak{g}^{*} \otimes S^{1} \mathfrak{g}^{*}$ and satisfies $\theta(X) \beta=i(X) d \beta$. The differential $d$ satisfies $\theta(X)=d i(X)+i(X) d$ for all $X \in \mathfrak{g}$.

If $\mathfrak{h}$ is a Lie subalgebra of $\mathfrak{g}$, we say that an element $\alpha \in W(\mathfrak{g})$ is $\mathfrak{h}$-basic if $\theta(X) \alpha=i(X) \alpha=0, \forall X \in \mathfrak{h}$. Define the relative Weil algebra $W(\mathfrak{g}, \mathfrak{h})$ to be the set of $\mathfrak{h}$-basic elements in $W(\mathfrak{g}) . W(\mathfrak{g}, \mathfrak{h})$ is a differential subalgebra of $W(\mathfrak{g})$. If $G$ is a Lie group with Lie algebra $\mathfrak{g}$ and $H \subset G$ is a subgroup with Lie algebra $\mathfrak{h} \subset \mathfrak{g}$, we say an element $\alpha \in W(\mathfrak{g})$ is $H$-basic if $\alpha$ is $\mathfrak{h}$-basic and Ad $h \cdot \alpha=\alpha, \forall h \in H$. Define $W(\mathfrak{g}, H)$ to be the set of $H$-basic elements of $W(\mathfrak{g}) ; W(\mathfrak{g}, H)$ is also a differential subalgebra of $W(\mathfrak{g})$. If $H$ is connected, $W(\mathfrak{g}, \mathfrak{h})=W(\mathfrak{g}, H)$.

Now let $\omega$ be a connection in a principal bundle $\pi: P \rightarrow M$ with structure group $G$; recall that $\omega$ is a $\mathfrak{g}$-valued 1 -form on $P$ and the curvature $\Omega$ is a $\mathfrak{g}$-valued 2-form. Define an algebra homomorphism $\kappa(\omega): W(\mathfrak{g}) \rightarrow \Omega(P)$ as follows: for $\alpha \in \Lambda^{1} \mathfrak{g}^{*}, \kappa(\omega)(\alpha)=\alpha \circ \omega$; for $\beta \in S^{1} \mathfrak{g}^{*}, \kappa(\omega)(\beta)=\beta \circ \Omega$. $\kappa(\omega)$ is a map of differential graded algebras, and in fact the differential $d$ on $W(\mathfrak{g})$ is the unique one making $\kappa(\omega)$ a differential map for all connections $\omega$. Furthermore, for $X \in \mathfrak{g}$ define $\bar{X}$ to be the vertical vector field on $P$ given by $\bar{X}_{u}=\left.\frac{d}{d t}\right|_{t=0} u \cdot \exp t X$. Then $\kappa(\omega) \circ i(X)=i(\bar{X}) \circ \kappa(\omega)$ and $\kappa(\omega) \circ \theta(X)=$ $\theta(\bar{X}) \circ \kappa(\omega)$ for all $X \in \mathfrak{g}$. For $\tau \in \Lambda \mathfrak{g}^{*}$ we abbreviate $\kappa(\omega)(\tau)=\tau(\omega)$; for $\sigma \in S \mathfrak{g}^{*}$ we write $\kappa(\omega)(\sigma)=\sigma(\Omega)$.

Let $K$ be a maximal compact subgroup of $G$. The restriction of $\kappa(\omega)$ to $W(\mathfrak{g}, K)$ takes values in the subalgebra $\Omega(P)_{K}=\{\alpha \in \Omega(P) \mid i(\bar{X}) \alpha=0, \forall X \in$ $\mathfrak{k} ; \alpha \cdot k=\alpha, \forall k \in K\} . \Omega(P)_{K}$ is naturally isomorphic to $\Omega(P / K)$, and $P / K \rightarrow M$ is a bundle with contractible fiber, so there is a smooth section $s: M \rightarrow P / K$ unique up to homotopy. The Chern-Weil homomorphism is the composition

$$
s^{*} \circ \kappa(\omega): W(\mathfrak{g}, K) \rightarrow \Omega(M) .
$$

This is a map of differential graded algebras and it therefore induces a map on cohomology,

$$
H\left(s^{*} \circ \kappa(\omega)\right): H^{*}(W(\mathfrak{g}, K)) \rightarrow H^{*}(\Omega(M)) .
$$


It is a standard result that $H\left(s^{*} \circ \kappa(\omega)\right)$ does not depend on the choices of $s$ and $\omega$ (since any two sections are homotopic and the set of connections is a convex subset of the affine space of $\mathfrak{g}$-valued 1-forms on $P$ ). For $\mathfrak{g}=\mathfrak{g l}(n)$ and $K=O(n)$, the image of the map $H\left(s^{*} \circ \kappa(\omega)\right)$ is the algebra of Pontryagin classes of $P$.

Now let $(M, \mathscr{F})$ be a codimension- $n, C^{2}$ foliation, $T \mathscr{F}$ the tangent bundle of $\mathscr{F}$, and $Q=T M / T \mathscr{F}$ the normal bundle of $\mathscr{F}$. Fix a Riemannian metric $g$ on $M$ and use it to identify $Q$ with the subbundle of $T M$ orthogonal to $T \mathscr{F}$; let $\pi_{Q}: T M \rightarrow Q$ be orthogonal projection. Let $\pi: P(Q) \rightarrow M$ be the principal $G L(n, \mathbf{R})$ bundle associated to $Q$. Let $\mathscr{I}=\mathscr{I}(\mathscr{F})$ be the differential ideal of forms vanishing on $T \mathscr{F}$.

Definition. Let $\omega$ be a connection on $P(Q)$ and $\nabla$ the associated covariant derivative on $Q . \omega$ is called a Bott connection if for all $X \in T \mathscr{F}$ and every section $Y$ of $Q, \nabla_{X} Y=\pi_{Q}[X, Y]$.

Bott connections always exist in the full frame bundle of the normal bundle [B2], and the set of such connections is convex. A Bott connection has the important property that the curvature of the connection vanishes when restricted to the leaves. Thus, if $c$ is an invariant homogeneous polynomial of degree $k$ on $\mathfrak{g l}(n)$ then $c(\Omega) \in \mathscr{I}^{k}$. Note that $\mathscr{I}$ is locally generated by $n$ independent 1 -forms, so $\mathscr{I}^{n+1}=0$. In particular, if the degree of $c$ is greater than $n$, then $c(\Omega)=0$. This is the Bott vanishing theorem [B2].

We now suppose $P$ is a principal subbundle of $P(Q)$ with structure group $G \subset G L(n, \mathbf{R})$ and we assume that $P$ admits a Bott connection $\omega$, i.e., there is a Bott connection on $P(Q)$, which restricts to a connection $\omega$ on $P$. This is equivalent to assuming that $P$ is a "holonomy invariant" reduction of $P(Q)$ (see below). Let $K$ be the maximal compact subgroup of $G$; we assume that $K=G \cap O(n)$ (we can always take a conjugate of $G$ for which this is true). By our preceding remarks, if $\alpha \in W^{p, 2 q}(\mathfrak{g})$ and $q>n$, then $\kappa(\omega)(\alpha)=0$. Let $F^{2 q} W(\mathfrak{g})=S^{2 q} \mathfrak{g}^{*} \cdot W(\mathfrak{g})$ and define $W(\mathfrak{g})_{n}=W(\mathfrak{g}) / F^{2 n+2} W(\mathfrak{g}) . W(\mathfrak{g})_{n}$ is a differential graded algebra and $\kappa(\omega)$ factors to a map $\kappa(\omega): W(\mathfrak{g})_{n} \rightarrow \Omega(P)$. If we apply the same construction to the subalgebra $W(\mathfrak{g}, K)$, we get a quotient $W(\mathfrak{g}, K)_{n}$ and a map $\kappa(\omega): \rightarrow W(\mathfrak{g}, K)_{n} \rightarrow \Omega(P / K)$; by composing with $s^{*}$ we get a map

$$
\Delta(\omega)=s^{*} \circ \kappa(\omega): W(\mathfrak{g}, K)_{n} \rightarrow \Omega(M) .
$$

The induced map $\Delta(\omega)_{*}: H^{\cdot}\left(W(\mathfrak{g}, K)_{n}\right) \rightarrow H^{\cdot}(M)$ is independent of the choice of Bott connection (and of $s$ ) and will be denoted $\Delta_{*}$. If $P=P(Q)$, then the image of $H^{\cdot}\left(W(\mathfrak{g l}(n), O(n))_{n}\right)$ by $\Delta_{*}$ is called the algebra of characteristic classes of $\mathscr{F}$.

The characteristic homomorphism $\Delta_{*}$ satisfies two important functorial properties: first, if $P^{\prime} \subset P$ is a reduction of $P$ to the group $G^{\prime} \subset G$ and $P^{\prime}$ admits a Bott connection, then the following diagram commutes: 


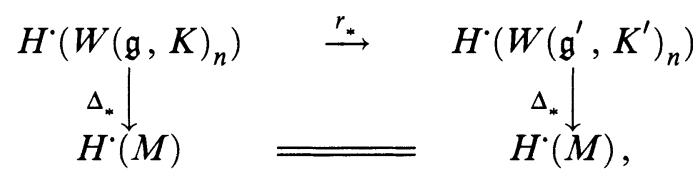

where $r_{*}$ is the map induced by the restriction map $r: \mathfrak{g}^{*} \rightarrow g^{\prime *}$. Thus the characteristic classes of the foliation can be viewed as obstructions to the existence of Bott connections in principal subbundles of the principal normal bundle of the foliation. In other words, these classes are obstructions to holonomy invariant reductions of $P(Q)$. Notice that if $P(Q)$ admits a holonomy invariant reduction to $O(n)$, then the algebra of characteristic classes of the foliation is exactly the algebra of Pontryagin classes of the normal bundle. The algebra of Pontryagin classes of $Q$ always appears as a subalgebra of $\Delta_{*}\left(H^{\cdot}\left(W(\mathfrak{g}, K)_{n}\right)\right)$.

$\Delta_{*}$ is also functorial with respect to transversal maps of foliations. If $(M, \mathscr{F})$ and $\left(M^{\prime}, \mathscr{F}^{\prime}\right)$ are codimension- $n$ foliated manifolds and $f: M \rightarrow M^{\prime}$ is a $C^{2}$ transversal mapping, then the normal bundle $Q$ of $\mathscr{F}$ is isomorphic to $f^{*} Q^{\prime}$, where $Q^{\prime}$ is the normal bundle of $\mathscr{F}^{\prime}$. Let $P^{\prime} \subset P\left(Q^{\prime}\right)$ be a subbundle with group $G \subset G L(n, \mathbf{R})$ and suppose that $P^{\prime}$ admits a Bott connection. There is a subbundle $P \subset P(Q)$ with group $G$ isomorphic to $f^{*} P^{\prime}$ and a bundle map $f_{*}: P \rightarrow P^{\prime}$ such that the following diagram commutes:

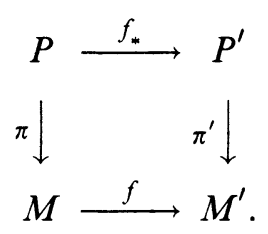

The pullback of the Bott connection on $P^{\prime}$ is a Bott connection on $P$ and the following diagram commutes:

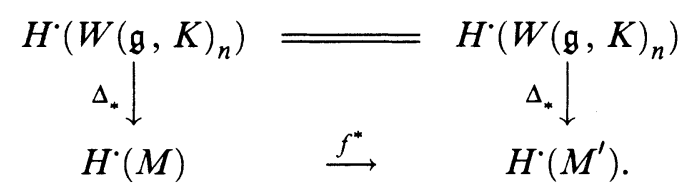

The algebra $W(\mathfrak{g l}(n), O(n))_{n}$ contains a subalgebra $W O_{n}$ whose cohomology is isomorphic to the cohomology of $W(\mathfrak{g l}(n), O(n))_{n}$. This subalgebra is obtained as follows: first note that $W(\mathfrak{g l}(n))$ is acyclic in positive dimensions. The set of $O(n)$-basic elements of $W(\mathfrak{g l}(n))$ is the polynomial algebra $\mathbf{R}\left[c_{1}, \ldots, c_{n}\right]$, where $c_{i}$ is the $i$ th Chern polynomial. The elements $c_{i}$ are closed and therefore exact. Choose $y_{i} \in W(\mathfrak{g l}(n))$ with $d y_{i}=c_{i}$. Let

$$
W_{n}=\bigwedge\left(y_{1}, y_{2}, \ldots, y_{n}\right) \otimes \mathbf{R}\left[c_{1}, \ldots, c_{n}\right]_{n},
$$

where $\mathbf{R}\left[c_{1}, \ldots, c_{n}\right]_{n}$ is the truncation of $\mathbf{R}\left[c_{1}, \ldots, c_{n}\right]$ in degrees greater than $2 n$ (recall that degree $\left(c_{i}\right)=2 i$ ). The next two theorems are classical (cf. [Ha]). 
Theorem 1.1. The inclusion $W_{n} \rightarrow W(\mathfrak{g l}(n))$ induces an isomorphism in cohomology.

For $i$ odd, it is possible to choose the elements $y_{i}$ in $W(\mathfrak{g l}(n), O(n))$. Define

$$
W O_{n}=\bigwedge\left(y_{1}, y_{3}, \ldots, y_{q}\right) \otimes \mathbf{R}\left[c_{1}, \ldots, c_{n}\right]_{n},
$$

where $q$ is the greatest odd integer less than or equal to $n$.

Theorem 1.2 [Ha]. The inclusion $W O_{n} \rightarrow W(\mathfrak{g l}(n), O(n))_{n}$ induces an isomorphism in cohomology.

In degree $\leq 2 n, H^{\cdot}\left(W O_{n}\right)$ is the algebra of Pontryagin classes. J. Vey computed the following basis of $H^{\cdot}\left(W O_{n}\right)$ in degree greater than $2 n$. Let $I=\left(i_{1}, \ldots, i_{k}\right)$ be a multi-index with $0<i_{1}<\cdots<i_{k} \leq m ; i_{j}$ odd. Let $J=\left(j_{1}, \ldots, j_{p}\right)$, with $1 \leq j_{1} \leq \cdots \leq j_{p} \leq n$ and set $|J|=\sum_{l=1}^{p} j_{l}$. Define

$$
y_{I} c_{J}=y_{i_{1}} \wedge \cdots \wedge y_{i_{k}} \otimes c_{j_{1}} \otimes \cdots \otimes c_{j_{p}} .
$$

The collection of all such $y_{I} c_{J}$ is a basis of $W O_{n}$. The subset

$$
\left\{y_{I} c_{J}|| J|\leq n ;| J \mid+i_{1}>n ; i_{1} \leq \text { any odd } j_{l}\right\}
$$

is a basis of $H^{\cdot}\left(W O_{n}\right)$. The classes in degree greater than $2 n$ with $|J|=n$ are called the residual classes. These classes have the property that their images under the characteristic homomorphism of a foliated manifold lie in the $n$th exterior power of the ideal of forms vanishing along the leaves.

We now define the Weil homomorphism. Let

$$
\Omega(M, \mathscr{F})=\mathscr{I}^{n} \text { and } \Omega_{c}(M, \mathscr{F})=\Omega(M, \mathscr{F}) \wedge \Omega_{c}(M),
$$

where $\Omega_{c}(M)$ is the space of compactly supported forms on $M . \Omega(M, \mathscr{F})$ is a differential ideal in $\Omega(M)$ and is therefore a differential subcomplex of the De Rham complex of $M$. We set

$$
\begin{aligned}
& H^{\cdot}(M, \mathscr{F})=H^{*}(\Omega(M, \mathscr{F}), d), \\
& H_{c}^{\cdot}(M, \mathscr{F})=H^{*}\left(\Omega_{c}(M, \mathscr{F}), d\right) .
\end{aligned}
$$

Denote by $\left(\bigwedge \mathfrak{g l}(n)^{*}\right)_{O(n)}$ the subalgebra of $W(\mathfrak{g l}(n), O(n))$ consisting of $O(n)$-basic elements of $\Lambda \mathfrak{g l}(n)^{*} .\left(\Lambda \mathfrak{g l}(n)^{*}\right)_{O(n)}$ is closed with respect to the Chevalley-Eilenberg differential $d_{\Lambda}$ and

$$
H^{\cdot}\left(\left(\bigwedge \mathfrak{g l}(n)^{*}\right)_{O(n)}, d_{\Lambda}\right)=H^{\cdot}(\mathfrak{g l}(n), O(n))
$$

by definition. Define a map

$$
\chi:\left(\bigwedge \mathfrak{g l}(n)^{*}\right)_{O(n)} \rightarrow \Omega_{c}(M, \mathscr{F})^{*}
$$

by $\chi(\tau)(\phi)=\int_{M} \Delta(\tau) \wedge \phi$. The map $\chi$ is a map of differential complexes, where we take the differential on $\Omega_{c}(M, \mathscr{F})^{*}$ to be the adjoint of the exterior derivative. Thus $\chi$ induces a map $\chi: H^{*}(\mathfrak{g l}(n), O(n)) \rightarrow H_{c}^{*}(M, \mathscr{F})^{*}$ which 
is independent of all choices. This map is called the Weil homomorphism. For each $\tau \in H^{k}(\mathfrak{g l}(n), O(n))$ we have $\chi(\tau): H_{c}^{m-k}(M, \mathscr{F}) \rightarrow \mathbf{R} ; \chi(\tau)$ is called the Weil operator associated to $\tau$.

Let $y_{I} c_{J}$ be an element of the Vey basis representing a residual characteristic class, and let $\tau_{I}$ be the purely exterior part of $y_{I}$. Then $\Delta_{*}\left(y_{I} c_{J}\right)=\Delta_{*}\left(\tau_{I} c_{J}\right)$. Moreover, $\Delta\left(c_{J}\right)$ is a well-defined (independent of the Bott connection) element of $H(M, \mathscr{F})$. Let $\phi \in \Omega_{c}^{k}(M)$ be a closed form, where $k=\operatorname{dim} M-\operatorname{deg} y_{I}$. Then $\Delta\left(c_{J}\right) \wedge \phi$ defines a class in $H_{c}(M, \mathscr{F})$ and

$$
\chi\left(\tau_{I}\right)\left[\Delta\left(c_{J}\right) \wedge \phi\right]=\int_{M} \Delta\left(\tau_{I}\right) \wedge \Delta\left(c_{J}\right) \wedge \phi=\left\langle\Delta_{*}\left(y_{I} c_{J}\right) \cup[\phi],[M]\right\rangle .
$$

By Poincare duality the class $\Delta_{*}\left(y_{I} c_{J}\right)$ is therefore determined completely by the map $\chi\left(\tau_{I}\right)$ and the class $\Delta_{*}\left(c_{J}\right) \in H(M, \mathscr{F})$. Note that the Weil homomorphism is functorial with respect to holonomy invariant reductions of the normal bundle. Thus if $P(Q)$ admits a holonomy invariant reduction to a group $H \subset G L(n, \mathbf{R})$, then the following diagram is defined and commutes:

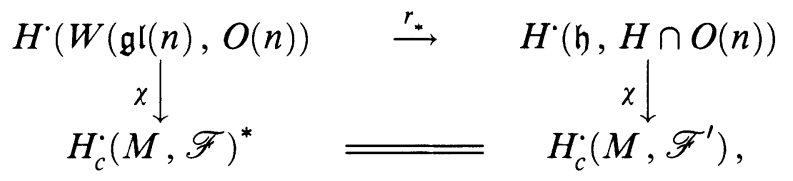

We stress that if $\tau_{I} \in H^{\cdot}(\mathfrak{g l}(n), O(n))$ restricts to zero in $H^{\cdot}(\mathfrak{h}, H \cap O(n))$ then every residual class $y_{I} c_{J}(\mathscr{F})$ vanishes.

We now give an overview of the proof of the Main Theorem. Let $\tau \in$ $H^{\cdot}(\mathfrak{g l}(n), O(n))$. The Weil operator $\chi(\tau)$ is given by integrating $\Delta(\tau)$ against forms $\phi$ which are "maximally transverse," i.e., lie in the $n$th exterior product of the ideal of differential forms vanishing along the leaves. Thus to compute $\int_{M} \Delta(\tau) \wedge \phi$ we need only know the values of $\Delta(\tau)$ restricted to $T \mathscr{F}$. This suggests that we might be able to compute $\chi(\tau)$ using a version of $\Delta(\tau)$, which is smooth along the leaves but only measurable transversally.

The hypothesis to the Main Theorem yields a principal $H$-subbundle $P^{\prime}$ of $P(Q)$ which is measurable, smooth along the leaves, and holonomy invariant. We show in $\S 5$, following [H-K], that the Bott connection makes sense along the leaves for this subbundle and takes values in the Lie algebra $\mathfrak{h}$ of $H$. By applying $\tau$ to the Bott connection, we get a measurable tangentially defined 1-form $\tau(\omega)$ on $P^{\prime}$. To get a measurable version of $\Delta(\tau)$ we construct a section of $P^{\prime} / K \rightarrow M$, which is measurable and smooth along the leaves, and use this section to pull back $\tau(\omega)$ along the leaves. It is in the construction of this section that we require that $H$ be reductive with compact center. Finally, we show, again following [H-K], that the measurable version of $\Delta(\tau)$ suffices to compute the Weil operator $\chi(\tau)$. For this we construct a uniformly bounded sequence of smooth forms converging to the measurable version of $\Delta(\tau)$; the proof follows by an application of the Lebesgue Dominated Convergence Theorem. 


\section{The holonomy COCYCle AND the MAIN Hypothesis}

We begin by establishing some notation that will be used throughout the rest of this chapter.

$M$ is an $m$-dimensional, orientable, $C^{2}$ manifold with a $C^{2}$, codimension $n$ foliation $\mathscr{F}, T \mathscr{F}$ is the tangent bundle of $\mathscr{F}$, and $Q=T M / T \mathscr{F}$ is the normal bundle of $\mathscr{F}$ which we identify with the orthogonal complement of $T \mathscr{F}$ in $T M$ relative to a Riemannian metric $g$ on $M$. Let $\pi_{Q}$ be the orthogonal projection of $T M$ onto $Q$. Let $P$ be the principal frame bundle of $Q$; the metric $g$ restricts to a metric $h$ on $Q$ and therefore determines a smooth section $s: M \rightarrow P / O(n)$.

Let $X=G L(n, \mathbf{R}) / O(n) . \quad X$ has a left invariant Riemannian metric of nonpositive sectional curvature. This metric induces a Riemannian metric on the fibers of the bundle

$$
P / O(n) \cong P \times_{G L(n, \mathbf{R})} X \stackrel{\pi}{\longrightarrow} M .
$$

For $x \in M$ let $\rho_{x}$ denote the associated distance function on the fiber $(P / O(n))_{x}$ $=\pi^{-1}(x)$. A connection $\omega$ yields an isomorphism $T_{u} P \rightarrow T_{\pi(u)} M \oplus \mathfrak{g l}(n)$ for each $u \in P$. We define a Riemannian metric on $P$ via the above isomorphism by giving $T_{\pi(u)} M$ the metric $g, \mathfrak{g l}(n)$ an $O(n)$-invariant metric compatible with the metric on $X$, and taking $T_{\pi(u)} P$ to be perpendicular to $\mathfrak{g l}(n)$. This metric on $P$ is invariant by the (right) action of $O(n)$ and therefore induces a Riemannian metric on $P / O(n)$.

Let $I$ denote the interval $(-1,1)$. A foliation chart for $\mathscr{F}$ consists of an open set $U \subset M$ and a diffeomorphism $\phi: U \rightarrow I^{m-n} \times I^{n}$ such that for each $x \in I^{n}$, the set $\phi^{-1}\left(I^{m-n} \times\{x\}\right)$ is a connected component of $L \cap U$ for some leaf $L$ of $\mathscr{F} \cdot \phi^{-1}\left(I^{m-n} \times\{x\}\right)$ is called a plaque. $\phi: U \rightarrow I^{m-n} \times I^{n}$ is a regular foliation chart if there is a foliation chart $\psi: V \rightarrow(-2,2)^{m-n} \times$ $(-2,2)^{n}$ such that $\bar{U} \subset V$ and $\psi \mid U=\phi$. We fix a simple locally finite covering $\left\{\left(U_{\alpha}, \phi_{\alpha}\right)\right\}_{\alpha \in A}$ of $M$ by regular foliation charts. Let $\left\{\lambda_{\alpha}\right\}_{\alpha \in A}$ be a partition of unity subordinate to $\left\{U_{\alpha}\right\}_{\alpha \in A}$.

Each regular foliation chart $\left(\phi_{\alpha}, U_{\alpha}\right)$ induces a trivialization $P \mid U_{\alpha} \rightarrow I^{m} \times$ $G L(n, \mathbf{R})$, as follows: an element $u \in P$ is an isomorphism $u: \mathbf{R}^{n} \rightarrow Q_{\pi(u)}$. If we fix the standard basis in $\mathbf{R}^{n}$ and choose a basis in $Q_{\pi(u)}$, we can write $u$ as an element of $G L(n, \mathbf{R})$. For $x \in U_{\alpha}$ we take as our basis of $Q_{x}$ the set $\left\{\pi_{Q}\left(\phi_{\alpha *}^{-1}\left(\partial / \partial x_{i}\right)\right)\right\}_{i=m-n+1}, \ldots, m$. Define a Bott connection $\omega$ on $P$ by first taking local connections $\omega_{\alpha}$ on $P \mid U_{\alpha}$ for which the trivialization is parallel (i.e., $\omega_{\alpha}$ is the product connection on $I^{m} \times G L(n, \mathbf{R})$ ) and then glueing these local connections together using the partition of unity. Since the transition functions for the local trivializations of $P$ are constant along the leaves, the connection $\omega=\sum_{\alpha \in A} \lambda_{\alpha} \omega_{\alpha}$ is flat along the leaves.

Let $\Phi_{\alpha}: U_{\alpha} \rightarrow I^{n}$ be the composition of $\phi_{\alpha}$ with the projection $I^{m-n} \times I^{n} \rightarrow$ $I^{n}$ and define $T_{\alpha}=I^{n}$. Let $T=\bigsqcup_{\alpha \in A} T_{\alpha}$. For each $\alpha$ there is a map 
$T_{\alpha} \rightarrow U_{\alpha}$ given by $x \mapsto \phi_{\alpha}^{-1}(0, x)$. This gives a map $\Psi: T \rightarrow M$, which is a local diffeomorphism onto its image; let $\widetilde{T}=\Psi(T) . \widetilde{T}$ is a complete transversal for $\mathscr{F}$, i.e., every leaf of $\mathscr{F}$ meets $\widetilde{T}$ in a countable, nonempty set.

Let $U_{\alpha \beta}=U_{\alpha} \cap U_{\beta}$ and $T_{\alpha \beta}=\Phi_{\beta}\left(U_{\alpha \beta}\right)$. Define $\gamma_{\alpha \beta}: T_{\alpha \beta} \rightarrow T_{\beta \alpha}$ by $\gamma_{\alpha \beta}(x)=\Phi_{\alpha}\left(\Phi_{\beta}^{-1}(x)\right)$. The collection $\left\{\gamma_{\alpha \beta} \mid U_{\alpha \beta} \neq \varnothing\right\}$ generates the holonomy pseudogroup $\mathscr{G}$ acting on $T$. Let $P T=T \times G L(n, \mathbf{R})$ be the principal frame bundle of $T$; the collection $\left\{D \gamma_{\alpha \beta} \mid U_{\alpha \beta} \neq \varnothing\right\}$ generates the linear holonomy pseudogroup $D \mathscr{G}$ acting on $P T$. We say that $P$ admits a holonomy invariant reduction to a group $H \subset G L(n, \mathbf{R})$ if there is a principal $H$ subbundle of $P T$ which is invariant by $D \mathscr{G}$. (We will see that any $D \mathscr{G}$-invariant subbundle of $P T \rightarrow T$ "pulls back" to a subbundle of $P \rightarrow M$.)

The orbits of the pseudogroup $\mathscr{G}$ yield an equivalence relation $R$ on $T$ : we have $(x, y) \in R$ if and only if $\Psi(x)$ and $\Psi(y)$ lie in the same leaf of $\mathscr{F}$. For $x \in T$ we let $R_{x} \subset T$ denote the equivalence class of $x$. Each equivalence class of $R$ has a natural metric induced from the word metric on $\mathscr{G}$; explicitly, for $(x, y) \in R, d(x, y)$ is the smallest integer $k$ such that $x=\gamma_{\alpha_{1} \beta_{1}} \circ \cdots \circ \gamma_{\alpha_{k} \beta_{k}}(y), \beta_{i}=\alpha_{i+1}$. Equivalently, $d(x, y)$ is the minimal number of plaques in a "plaque path" from $x$ to $y$.

A cocycle over $R$ is a measurable map $\nu: R \rightarrow G L(n, \mathbf{R})$ satisfying the cocycle identity

$$
\nu(x, y) \nu(y, z)=\nu(x, z) \quad \text { for all } y, z \in R_{x},
$$

almost everywhere (see [F-M, pp. 304-305]). Two cocycles $\nu, \delta$ are equivalent (or cohomologous) if there is a measurable map $f: T \rightarrow G L(n, \mathbf{R})$ such that $\nu(x, y)=f(x)^{-1} \delta(x, y) f(y)$ almost everywhere. The map $f$ is called a coboundary from $\delta$ to $\nu$. Let $R^{1}=\{(x, y) \in R \mid d(x, y)=1\}$; a cocycle $\nu$ is locally tempered if for every compact set $K \subset M, \nu \mid R^{1} \cap \Psi^{-1}(K)^{2}$ is essentially bounded.

Define a cocycle $\nu: R \rightarrow G L(n, \mathbf{R})$ as follows: for $(x, y) \in R, x \neq y$, choose $\gamma \in \mathscr{G}$ with $y=\gamma(x)$. Let $\nu(x, y)=D \gamma_{x}$, i.e., the differential of $\gamma$ evaluated at $x \in T$. The following lemma shows that $\nu$ is well defined.

Lemma $2.1[\mathrm{H}-\mathrm{K}]$. Almost every leaf has trivial linear holonomy.

Thus for almost every $x \in T, \gamma(x)=x$ implies that $D_{\gamma_{x}}$ is the identity matrix. It follows that $\nu$ satisfies the cocycle identity almost everywhere. Note also that $\nu$ is locally tempered because the foliation charts are regular and the covering is locally finite. We now state the Main Hypothesis from which we will derive information about the vanishing of certain residual characteristic classes of $\mathscr{F}$.

Main Hypothesis. $\nu$ is measurably equivalent to a locally tempered cocycle $\delta$ taking values (essentially) in a closed subgroup $H \subset G L(n, \mathbf{R})$ which is stable by some Cartan involution of $G L(n, \mathbf{R})$. 
We can assume without loss of generality that $H$ is stable by the standard Cartan involution of inverse transpose on $G L(n, \mathbf{R})$, so that the maximal compact subgroup of $H$ is $K=H \cap O(n)$. The importance of the assumption on $H$ is that the submanifold $H / K \rightarrow X$ is totally geodesic (see $\S 7$ ). The importance of $\delta$ being tempered will become apparent below.

Let $f: T \rightarrow G L(n, \mathbf{R})$ be the coboundary from $\nu$ to $\delta$, so $\delta(x, y)=$ $f(x)^{-1} \nu(x, y) f(y)$ and $\delta$ (essentially) takes values in $H$. If we consider $f$ as a section $T \rightarrow P T$, then the (right) $H$-orbit of $f$ determines a $D \mathscr{G}$-invariant measurable principal $H$-subbundle $P^{\prime} T$ of $P T$. We define a subbundle $P^{\prime} \subset P$ as follows: the map $\Phi_{\alpha}: U_{\alpha} \rightarrow T_{\alpha}$ induces a bundle map $\left(\Phi_{\alpha}\right)_{*}: P\left|U_{\alpha} \rightarrow P T\right| T_{\alpha}$. We take $P^{\prime} \mid U_{\alpha}$ to be the inverse image of $P^{\prime} T \mid T_{\alpha}$ under this map. It is easy to see that $P^{\prime} \mid U_{\alpha}$ is canonically isomorphic to the pullback $\Phi_{\alpha}^{*}\left(P^{\prime} T \mid T_{\alpha}\right)$. The holonomy invariance of $P^{\prime} T$ guarantees that the local bundles $P^{\prime} \mid U_{\alpha}$ fit together to give a global principal $H$-bundle $P^{\prime} \rightarrow M$. This bundle is measurable and smooth along the leaves, i.e., $P^{\prime}$ admits local sections which (as maps into $P$ ) are measurable and smooth along the leaves. The quotient bundle $P^{\prime} / K \rightarrow M$ has the important property that each fiber $\left(P^{\prime} / K\right)_{x}$ is a totally geodesic submanifold of $(P / O(n))_{x}$.

\section{NONLINEAR AVERAGING}

It will be necessary at several points in the proof of the Main Theorem to construct global sections of $P / O(n) \rightarrow M$ by piecing together local sections. In this section we introduce a suitable technique for doing this. We refer the reader to a paper of Karcher [K] for proofs of some of the resuits in this section.

Recall that each fiber of $P / O(n) \rightarrow M$ has a metric of nonpositive sectional curvature. Let $\left\{t_{\alpha}: U_{\alpha} \rightarrow P / O(n) \mid U_{\alpha}\right\}$ be a collection of local sections. For $z \in M$ define a function $P_{z}$ on $(P / O(n))_{z}$ by

$$
P_{z}(x)=\sum_{\alpha} \lambda_{\alpha}(z) \rho_{z}\left(x, t_{\alpha}(z)\right)^{2} .
$$

Then $\operatorname{grad} P_{z}(x)=-\sum_{\alpha} \lambda_{\alpha}(z) \exp _{x}^{-1} t_{\alpha}(z)$ [K]. It can be shown that $\operatorname{grad} P_{z}(x)$ has unique zero $m_{z}$, and that $m_{z}$ is the unique minimum of the function $P_{z}(x)$. We write $m_{z}=\sum \lambda_{\alpha}(z) t_{\alpha}(z)$. Define a global section $t: M \rightarrow P / O(n)$ by $t(z)=\sum \lambda_{\alpha}(z) t_{\alpha}(z)$.

Example. Consider the special case of $G L(2, \mathbf{R}) / O(2)$. Let $\sigma=\overline{\left(\begin{array}{ll}2 & 0 \\ 0 & \frac{1}{2}\end{array}\right)}$ and $\tau=\overline{\left(\begin{array}{ll}1 & 0 \\ 0 & 1\end{array}\right)}$, where the bar denotes a coset of $O(2)$. We will compute

$$
m=\frac{1}{2} \tau+\frac{1}{2} \sigma
$$

The point $m$ is the unique minimum of the function $P(x)=\frac{1}{2} \rho(x, \tau)^{2}+$ $\frac{1}{2} \rho(x, \sigma)^{2}$. Clearly $m$ will be the midpoint of the geodesic segment connecting $\tau$ to $\sigma$. This geodesic is given by

$$
t \rightarrow \overline{\left(\begin{array}{cc}
e^{t} & 0 \\
0 & e^{-t}
\end{array}\right)}
$$


and by an easy computation we see that

$$
m=\overline{\left(\begin{array}{cc}
\sqrt{2} & 0 \\
0 & \frac{\sqrt{2}}{2}
\end{array}\right) .}
$$

Notice that both $\sigma$ and $\tau$ are in the totally geodesic subspace $S L(2, \mathbf{R}) / S O(2)$ and so is their average $m$.

The basic facts we require about this averaging procedure are

1. The averaging is invariant by isometries.

2. If the points $t_{\alpha}(z)$ all lie in a totally geodesic subspace of $(P / O(n))_{z}$, then $\sum \lambda_{\alpha}(z) t_{\alpha}(z)$ lies in the same totally geodesic subspace.

3. If the sections $t_{\alpha}$ are smooth, then the global section $t$ is smooth.

4. If the sections $t_{\alpha}$ are smooth, have bounded first derivatives, and lie within a uniformly bounded distance of the fixed section $s$, then the global section $t$ has bounded first derivatives. In fact, the derivatives of $t$ are bounded in terms of the derivatives of $t_{\alpha}$, the derivatives of $\lambda_{\alpha}$, and the (fiberwise) distances of $t_{\alpha}$ from $s$.

Remark. Hurder and Katok [H-K] use a different averaging technique to piece together local sections. Their technique consists of taking a linear average in the convex cone $S(n)$ of positive definite symmetric matrices, which is naturally identified with $G L(n, \mathbf{R}) / O(n)$. This technique is inappropriate for our purposes, because in general $H / H \cap K$ is not a convex subset of $S(n)$. Nevertheless, Hurder and Katok do state and give a proof of a weaker version of the Main Theorem for the case $H=S L(n, \mathbf{R})$. Their proof has a mistake in statement (7.25), although this error can be fixed by interpreting all multiplications to be not matrix multiplication, but the action of $G L(n, \mathbf{R})$ on $S(n)$; the appropriate local sections are then the positive square roots of the matrices defined in (7.25).

In the next section we show that the residual characteristic classes can be computed from "nice" measurable sections of $P / O(n)$. In $\S 5$ we construct a sequence of nice measurable sections of $P / O(n)$ converging to a measurable section of $P^{\prime} / K$.

\section{COMPUTING THE CHARACTERISTIC CLASSES FROM}

A MEASURABLE SECTION

A measurable section $t: M \rightarrow P / O(n)$ is bounded if

$$
\underset{x \in M}{\operatorname{ess} \sup _{x}} \rho_{x}(t(x), s(x))<\infty \text {. }
$$

A measurable section $t: P / O(n)$ is smooth $\left(C^{k}\right)$ along the leaves or tangentially smooth $\left(C^{k}\right)$ if the restriction of $t$ to any plaque of a foliation chart is smooth $\left(C^{k}\right)$. A tangentially $C^{1}$ section is said to be tangentially $C^{1}$ bounded if it is bounded and $\operatorname{ess} \sup _{v \in T^{1} \mathscr{F}}\left\|t_{*} v\right\|<\infty$, where $T^{1} \mathscr{F}$ is the bundle of unit 
vectors tangent to $\mathscr{F}, t_{*}$ denotes the leafwise differential of $t$, and $\left\|t_{*} v\right\|$ is the length of $t_{*} v$ in the metric on $P / O(n)$.

Suppose we are given a measurable section $t: M \rightarrow P / O(n)$, which is smooth along the leaves and tangentially $C^{1}$ bounded. We will construct a sequence of smooth sections $t_{i}: M \rightarrow P / O(n)$ such that $t_{i}(x) \rightarrow t(x)$ for almost every $x \in M$ and $t_{i *} \rightarrow t_{*}$ almost everywhere. We construct the sections $t_{i}$ by first constructing local sections over foliation charts and then piecing the local sections together to get a global section.

The local trivializations of $P$ induce local trivializations $P / O(n) \mid U_{\alpha} \rightarrow$ $I^{m} \times X$, and via these trivializations $t$ yields functions $t_{\alpha}: I^{m-n} \times I^{n} \rightarrow X$ which are measurable, bounded, and smooth along the plaques. Let $x_{0}$ be the identity coset in $X$; since $X$ has nonnegative sectional curvature, exp: $T_{x_{0}} X \rightarrow X$ is a diffeomorphism. Fix an isomorphism of $T_{x_{0}} X$ with $\mathbf{R}^{q}$ and define $\widetilde{t}_{\alpha}: I^{m} \rightarrow \mathbf{R}^{q}$ by $\tilde{t}_{\alpha}(x)=\exp _{x_{0}}^{-1} t_{\alpha}(x)$. Then $\tilde{t}_{\alpha}$ is essentially bounded, measurable, and smooth along the plaques, and the derivatives of $\tilde{t}_{\alpha}$ along the plaques are essentially bounded.

Let $\left\{k_{i}\right\}$ be a sequence of smooth kernels on $I^{n} \times I^{n}$ with support converging uniformly to the diagonal. We denote a point of $I^{m}$ by $z=(x, y)$ where $x \in I^{m-n}, y \in I^{n}$. Define $\tilde{t}_{\alpha}^{i}: g I^{m} \rightarrow \mathbf{R}^{q}$ by

$$
\tilde{t}_{\alpha}^{i}(x, y)=\int_{I^{n}} \tilde{t}_{\alpha}(x, w) k_{i}(w, y) d w .
$$

Then $\tilde{t}_{\alpha}^{i} \rightarrow \widetilde{t}_{\alpha}$ in measure and $\left(\tilde{t}_{\alpha}^{i}\right)_{*} \rightarrow\left(\tilde{t}_{\alpha}\right)_{*}$ in measure with respect to the norm on linear maps $\mathbf{R}^{m-n} \rightarrow \mathbf{R}^{q}$. In fact,

$$
\left(\widetilde{t}_{\alpha}^{i}\right)_{*}\left(\partial / \partial x_{i}\right)=\int_{I^{n}}\left(\partial / \partial x_{i}\right) \tilde{t}_{\alpha}^{i}(x, w) k_{i}(w, y) d w,
$$

so $\mu\left\{(x, y) \mid\left\|\left(\tilde{t}_{\alpha}^{j}\right)_{*}\left(\partial / \partial x_{i}\right)_{(x, y)}-\left(t_{\alpha}^{j}\right)_{*}\left(\partial / \partial x_{i}\right)_{(x, y)}\right\|>\varepsilon\right\} \rightarrow 0$ as $j \rightarrow \infty$ for all $\varepsilon>0$. Thus

$$
\mu\left\{(x, y) \mid\left\|\left(\tilde{t}_{\alpha}^{j}\right)^{*}-\left(\tilde{t}_{\alpha}^{j}\right)_{*}\right\|_{(x, y)}>\varepsilon\right\} \rightarrow 0 \quad \text { as } j \rightarrow \infty, \forall \varepsilon>0 .
$$

Now by passing to a subsequence we may assume that $\tilde{t}_{\alpha}^{i} \rightarrow \widetilde{t}_{\alpha}$ almost everywhere and $\left(\tilde{t}_{\alpha}^{i}\right)_{*} \rightarrow\left(\tilde{t}_{\alpha}\right)_{*}$ almost everywhere.

Define $t_{\alpha}^{i}: I^{m} \rightarrow X$ by $t_{\alpha}^{i}(z)=\exp _{x_{0}} \tilde{t}_{\alpha}^{i}(z)$. Via our trivialization of $P / O(n) \mid U_{\alpha}$ we get smooth sections $t_{\alpha}^{i}: U_{\alpha} \rightarrow P / O(n) \mid U_{\alpha}$. These sections have the property that $t_{\alpha}^{i}(z) \rightarrow t_{\alpha}(z)$ for a.e. $z \in U_{\alpha}$ with respect to the metrics on the fibers of $P / O(n)$, and $\left(t_{\alpha}^{i}\right)_{*} \rightarrow\left(t_{\alpha}\right)_{*}$ almost everywhere.

For each $i$, the collection $\left\{t_{\alpha}^{i}\right\}_{\alpha \in A}$ is a collection of local smooth sections of $P / O(n)$, but of course these local sections need not agree on the overlaps. Define $t^{i}: M \rightarrow P / O(n)$ by $t^{i}(z)=\sum \lambda_{\alpha}(z) t_{\alpha}^{i}(z)$. 
Now fix an element $y_{I} c_{J}$ of the Vey basis representing a residual characteristic class and let $\tau_{I}$ be the purely exterior component of $y_{I}$. Let $k$ be the degree of $y_{I}$. Let $t: M \rightarrow P / O(n)$ be a measurable section smooth along the leaves and tangentially $C^{1}$ bounded. We now define a linear map

$$
\Lambda=\Lambda_{\tau_{I}}: \Omega_{c}^{m-k}(M, \mathscr{F}) \rightarrow \mathbf{R}
$$

Let $\phi \in \Omega_{c}^{m-k}(M, \mathscr{F})$ and define a measurable $m$-form $\Lambda_{\phi}$ as follows: fix $x \in M$ and let $Y_{1}, \ldots, Y_{m}$ be a basis of $T_{x} M$ such that $Y_{1}, \ldots, Y_{m-n}$ are tangent to $\mathscr{F}$. Define

$$
\begin{aligned}
& \Lambda_{\phi}\left(Y_{i_{1}}, \ldots, Y_{i_{k-n}}, Y_{m-n+1}, \ldots, Y_{m}\right) \\
& =c \cdot \sum_{\sigma \in S_{m-n}}(-1)^{\sigma} \tau_{I}(\omega)\left(t_{*} Y_{\sigma(1)}, \ldots, t_{*} Y_{\sigma(k)}\right) \\
& \cdot \phi\left(Y_{\sigma(k+1)}, \ldots, Y_{\sigma(m-n)}, Y_{m-n+1}, \ldots, Y_{m}\right)
\end{aligned}
$$

where $c=1 /\left(\left|S_{k}\right| \cdot\left|S_{m-n-k}\right|\right) . \quad \Lambda_{\phi}$ is a measurable, essentially bounded, compactly supported $m$-form on $M$, in particular, $\Lambda_{\phi}$ is integrable. Define $\Lambda(\phi)=\int_{M} \Lambda_{\phi}$.

Proposition 4.1. $\Lambda$ vanishes on closed forms. As an element of $H_{c}^{m-k}(M, \mathscr{F})^{*}$, $\Lambda$ coincides with $\chi\left(\tau_{I}\right)$.

Proof. Let $\phi=d \eta, \eta \in \Omega_{c}^{m-k-1}(M, \mathscr{F})$. Set $\alpha=t^{*} \tau_{I}(\omega) ;$ then

$$
\Lambda(\phi)=\int_{M} \alpha \wedge \phi=\int_{M} \alpha \wedge d \eta
$$

Define $\bar{d}(\alpha \wedge \eta)=d_{\mathscr{F}} \alpha \wedge \eta+(-1)^{\operatorname{deg} y_{I}} \alpha \wedge d \eta$. Notice that if $t$ is a smooth section, then $\bar{d}(\alpha \wedge \eta)=d(\alpha \wedge \eta)$ since $\eta \in \mathscr{I}^{n}$. We claim that $\int_{M} \bar{d}(\alpha \wedge \eta)=0$ and that $d_{\mathscr{F}} \alpha \wedge \eta=0$, and therefore that $\Lambda(\phi)=0$. The first claim follows from the leafwise Stokes theorem of $[\mathrm{H}-\mathrm{H}]$. The second claim follows from the fact that $d \tau_{I}(\omega) \in \pi^{*} \mathscr{I}$ so $d \tau_{I}(\omega)\left(t_{*} Y_{\sigma(1)}, \ldots, t_{*} Y_{\sigma(m-k+1)}\right)=0$ since $Y_{\sigma(1)}, \ldots, Y_{\sigma(m-k+1)} \in T_{x} \mathscr{F}$. Thus $\Lambda$ vanishes on exact forms.

To prove the second part of the theorem we use the sequence $\left\{t^{i}\right\}$ of smooth sections converging to $t$ which we constructed above. Define $\alpha_{i}=t^{i *} \kappa(\omega)\left(\tau_{I}\right)$. $\alpha_{i}$ is a smooth $k$-form and defines a linear map $\Lambda_{i}: H_{c}^{m-k}(M, \mathscr{F}) \rightarrow \mathbf{R}$. We will show that $\Lambda=\Lambda_{i}=\chi\left(y_{I}\right)$. 
Fix $\phi \in \Omega_{c}^{m-k}(M)$ with $d \phi=0$ and let $Y_{1}, \ldots, Y_{m}$ be an orthonormal basis of $T_{x} M$ such that $Y_{1}, \ldots, Y_{m-n}$ are tangent to $\mathscr{F}$. Then

$$
\begin{aligned}
& \left|\left(\Lambda_{i} \wedge \phi-\Lambda \wedge \phi\right)\left(Y_{1}, \ldots, Y_{m}\right)\right|=\left|\left(\alpha_{i}-\alpha\right) \wedge \phi\left(Y_{1}, \ldots, Y_{m}\right)\right| \\
& \quad \leq c \cdot \sum_{\sigma \in S_{m-n}}\left|\left(\alpha_{i}-\alpha\right)\left(t_{*} Y_{\sigma(1)}, \ldots, t_{*} Y_{\sigma(k)}\right)\right| \\
& \quad \cdot\left|\phi\left(Y_{\sigma(k+1)}, \ldots, Y_{\sigma(m-n)}, Y_{m-n+1}, \ldots, Y_{m}\right)\right| \\
& \quad \leq\|\phi\| \cdot \sum_{\sigma \in S_{m-n}}\left|\left(\alpha_{i}-\alpha\right)\left(Y_{\sigma(1)}, \ldots, Y_{\sigma(k)}\right)\right| \\
& \quad=\|\phi\| \cdot \sum_{\sigma \in S_{m-n}}\left|\tau_{I}(\omega)\left(\left(t_{*}^{i}-t_{*}\right) Y_{\sigma(1)}, \ldots,\left(t_{*}^{i}-t_{*}\right) Y_{\sigma(m-k)}\right)\right| .
\end{aligned}
$$

Since $t_{*}^{i} \rightarrow t_{*}$ almost everywhere, it follows that $\Lambda_{i} \wedge \phi \rightarrow \Lambda \wedge \phi$ almost everywhere. Moreover, the tangential $C^{1}$ boundedness of $t$ implies that the $\Lambda_{i}$ are uniformly tangentially $C^{1}$ bounded and therefore that the $\Lambda_{i} \wedge \phi$ are uniformly bounded. Thus by the dominated convergence theorem we conclude that $\Lambda_{i}(\phi) \rightarrow \Lambda(\phi)$. The lemma now follows since all the maps $\Lambda_{i}$ are smoothly homotopic and therefore coincide with $\chi\left(\tau_{I}\right)$ as elements of $H_{c}(M, \mathscr{F})^{*}$.

\section{TEMPERING THE COBOUNDARY}

In this section we construct a sequence of tangentially $C^{1}$-bounded measurable sections of $P / O(n) \rightarrow M$ which converge pointwise to a section of $P^{\prime} / K \rightarrow M$. In the next section we use this sequence to draw our conclusions about the residual characteristic classes of $\mathscr{F}$. We begin by constructing the section of $P^{\prime} / K \rightarrow M$. The coboundary $f: T \rightarrow G L(n, \mathbf{R})$, considered as a section $f: T \rightarrow P^{\prime} T$, yields local sections $f_{\alpha}: U_{\alpha} \rightarrow P^{\prime} \mid U_{\alpha}$ as follows: recall that $P^{\prime} \mid U_{\alpha}=\left(\Phi_{\alpha}\right)_{*}^{-1}\left(P^{\prime} T \mid T_{\alpha}\right)$, where $\left(\Phi_{\alpha}\right)_{*}: P\left|U_{\alpha} \rightarrow P T\right| T_{\alpha}$ is the differential of $\Phi_{\alpha}$. The map $\left(\Phi_{\alpha}\right)_{*}$ is injective on the fibers of $P \mid U_{\alpha}$ so we can take $f_{\alpha}(x)$ to be the unique element of $P_{x}^{\prime}$ satisfying $\left(\Phi_{\alpha}\right)_{*}\left(f_{\alpha}(x)\right)=f\left(\Phi_{\alpha}(x)\right)$. Note that $f_{\alpha}$ is smooth along the leaves and that for $x \in U_{\alpha \beta}, f_{\alpha}(x)$ and $f_{\beta}(x)$ differ by an element of $H$. This last statement follows easily from the identity

$$
\delta\left(x, \gamma_{\alpha \beta}(x)\right)=f(x)^{-1} \nu\left(x, \gamma_{\alpha \beta}(x)\right) f\left(\gamma_{\alpha \beta}(x)\right),
$$

since $\nu\left(x, \gamma_{\alpha \beta}(x)\right)$ is the transition function for local coordinates on $P$ and $\delta$ takes values in $H$. Let $\bar{f}_{\alpha}: U_{\alpha} \rightarrow P^{\prime} / K \mid U_{\alpha}$ be the composition of $f_{\alpha}$ with the quotient map. Now construct a global section $F: M \rightarrow P^{\prime} / K$ by setting $F(x)=\sum_{\alpha \in A} \lambda_{\alpha}(x) \bar{f}_{\alpha}(x)$, where we consider $\bar{f}_{\alpha}$ to be a section of $P / O(n) \mid U_{\alpha}$ and we use the averaging described in $\S 3$. The fact that $F$ takes values in $P^{\prime} / K$ follows from the total geodesity of $H / K$ in $X$. The section $F$ is smooth along the leaves, but it is not necessarily bounded, so we cannot use the results of $\S 4$ directly to compute the residual characteristic classes. To solve this problem we construct a sequence of tangentially $C^{1}$-bounded sections converging to $F$. 
Let $\bar{f}: T \rightarrow X$ be the projection of $f$ to the quotient and let $o$ denote the identity coset in $X$. For an element $x \in X$ define $|x|_{\rho}=\rho(o, x)$. For each integer $i>0$ define the $i$-truncation of $\bar{f}$ to be

$$
\bar{f}^{i}(x)=\left\{\begin{array}{l}
\bar{f}(x), \quad \text { if }|\bar{f}(x)|_{\rho} \leq i \\
\text { the point at distance } i \text { from } o \\
\text { along the geodesic from } o \text { to } \bar{f}(x), \text { otherwise. }
\end{array}\right.
$$

The section $\bar{f}^{i}: T \rightarrow P T / O(n)$ pulls back to give local sections $\bar{f}_{\alpha}^{i}: U_{\alpha} \rightarrow$ $P / O(n) \mid U_{\alpha}$. Define

$$
F^{i}(x)=\sum_{\alpha \in A} \lambda_{\alpha}(x) \bar{f}_{\alpha}^{i}(x)
$$

Clearly $F^{i}: M \rightarrow P / O(n)$ is a bounded, tangentially smooth action of $P / O(n)$, and $F^{i}$ converges to $F$ pointwise. It is not yet clear that $F^{i}$ is tangentially $C^{1}$-bounded.

The Cartan involution on $G L(n, \mathbf{R})$ yields decompositions of $\mathfrak{h}$ and $\mathfrak{g l}(n)$ into \pm 1 eigenspaces: $\mathfrak{h}=\mathfrak{p} \oplus \mathfrak{k} ; \mathfrak{g l}(n)=\mathfrak{p}(n) \oplus \mathfrak{o}(n)$. Consider the diagram

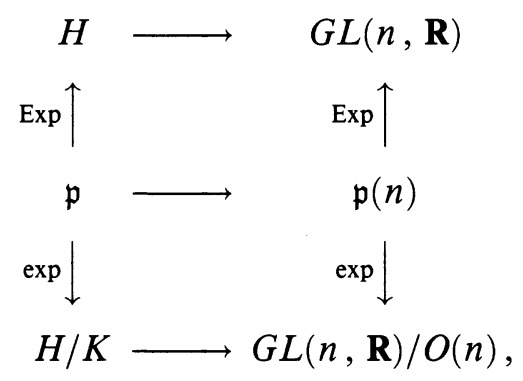

where Exp denotes the Lie algebra exponential map and exp is the metric exponential at the identity coset; the horizontal maps are all inclusions. This diagram commutes and the composition $\theta=\operatorname{Exp} \circ \exp ^{-1}$ gives a section of $H \rightarrow H / K$ (respectively, $G L(n, \mathbf{R}) \rightarrow X)$. Define $f^{i}=\theta \circ \bar{f}^{i}$ and set

$$
\delta^{i}(x, y)=f^{i}(x)^{-1} \nu(x, y) f^{i}(y)
$$

Fix $x \in M$ and choose $\alpha$ with $x \in U_{\alpha}$. We will compute the tangential derivative of $F^{i}$ in the local coordinates $\left(\phi_{\alpha}\right)_{*}: P / O(n) \mid U_{\alpha} \rightarrow U_{\alpha} \times X$. If $x \in U_{\beta}$ set $x_{\beta}=\Phi_{\beta}(x) \in T_{\beta}$; by definition, for $x \in U_{\alpha} \cap U_{\beta}, x_{\alpha}=\gamma_{\alpha \beta}\left(x_{\beta}\right)$. 
In local coordinates,

$$
\begin{aligned}
\left(\phi_{\alpha}\right)_{*} F^{i}(x) & =\sum_{\beta \in A} \lambda_{\beta}(x)\left(\phi_{\alpha}\right)_{*} \bar{f}_{\beta}^{i}(x) \\
& =\sum_{\beta \in A} \lambda_{\beta}(x)\left(\phi_{\alpha}\right)_{*}\left(\phi_{\beta}\right)_{*}^{-1}\left(\phi_{\beta}\right)_{*} \bar{f}_{\beta}^{i}(x) \\
& =\sum_{\beta \in A} \lambda_{\beta}(x)\left(\gamma_{\alpha \beta}\right)_{*} \bar{f}^{i}\left(\Phi_{\beta}(x)\right) \\
& =\sum_{\beta \in A} \lambda_{\beta}(x) \nu\left(x_{\beta}, x_{\alpha}\right) \bar{f}^{i}\left(x_{\beta}\right) \\
& =\sum_{\beta \in A} \lambda_{\beta}(x) f^{i}\left(x_{\alpha}\right) \bar{\delta}^{i}\left(x_{\beta}, x_{\alpha}\right) \\
& =f^{i}\left(x_{\alpha}\right) \sum_{\beta \in A} \lambda_{\beta}(x) \bar{\delta}^{i}\left(x_{\beta}, x_{\alpha}\right) .
\end{aligned}
$$

The second to last equality holds because $f^{i}$ is a coboundary from $\nu$ to $\delta^{i}$; the last equality holds because the center of gravity construction is invariant by translation. The functions $x \mapsto x_{\alpha}$ are (locally) constant along the leaves so the tangential derivative of $F^{i}$ depends only on the tangential derivatives of the $\lambda_{\beta}$ 's and $\left|\bar{\delta}^{i}\left(x_{\alpha}, x_{\beta}\right)\right|_{\rho}$. The following shows that the latter quantity is uniformly bounded on compact sets independent of $i$.

Lemma $5.1[\mathrm{H}-\mathrm{K}]$. $\left|\bar{\delta}^{i}\left(x_{\beta}, x_{\alpha}\right)\right|_{\rho} \leq 3\left\{\left|\bar{\nu}\left(x_{\beta}, x_{\alpha}\right)\right|_{\rho}+\left|\bar{\delta}\left(x_{\beta}, x_{\alpha}\right)\right|_{\rho}\right\}$ for all $x \in$ $U_{\alpha} \cap U_{\beta}$.

It follows that $\left|\bar{\delta}^{i}\left(x_{\alpha}, x_{\beta}\right)\right|_{\rho}$ is uniformly bounded on compact sets because $d\left(x_{\alpha}, x_{\beta}\right)=1$ and $\nu$ and $\delta$ are locally tempered.

We are now prepared to prove

Lemma 5.2. There is a sequence of tangentially smooth, tangentially $C^{1}$-bounded sections $F^{i}$ of $P / O(n) \rightarrow M$ which converges pointwise to $F$. Moreover, the tangential derivatives of the $F^{i}$ are uniformly bounded on compact sets (independent of $i$ ), and the tangential derivatives of the $F^{i}$ converge to the tangential derivatives of $F$.

Proof. We have established everything save the last statement for the sequence $\left\{F^{i}\right\}$ defined above. Fix $x \in M$ and choose $N>0$ such that $F^{i}(x)=F(x)$ for $i \geq N$. Then by the construction of $F^{i}$, and the fact that both $F^{i}$ and $F$ are smooth along the leaves, there is an open neighborhood $U$ of $x$ in the leaf through $x$ such that $F^{i}(y)=F(y)$ for all $y \in U$ and $i>N$.

Note that we are not claiming that the $F^{i}$ are uniformly bounded, only that the tangential derivatives are.

Corollary 5.3. The measurable tangential form $F^{*} \tau_{I}(\omega)$ is bounded and therefore defines a map $\Lambda_{F}: \Omega_{c}^{m-k}(M, \mathscr{F}) \rightarrow \mathbf{R}$. This map vanishes on closed forms and as an element of $H_{c}^{m-k}(M, \mathscr{F})^{*}, \Lambda_{F}$ coincides with $\chi\left(\tau_{I}\right)$. 
Proof. By Lemma 4.1, the corollary holds if we replace $F$ by $F^{i}$. But $\left(F^{i}\right)^{*} \tau_{I}(\omega)$ converges uniformly on compact subsets of $T \mathscr{F}$ to $F^{*} \tau_{I}(\omega)$. Therefore by the dominated convergence theorem, for $\phi \in \Omega_{c}^{m-k}(M, \mathscr{F})$,

$$
\Lambda_{F}(\phi)=\int F^{*} \tau_{I}(\omega) \wedge \phi=\lim \int\left(F^{i}\right)^{*} \tau_{I}(\omega) \wedge \phi=\lim \Lambda_{F^{i}}(\phi) .
$$

But the operator $\Lambda_{F^{i}}$ vanishes on closed forms and $\Lambda_{F^{i}}=\chi\left(\tau_{I}\right)$ as elements of $H_{c}^{m-k}(M, \mathscr{F})^{*}$. Thus $\Lambda_{F}=\chi\left(\tau_{I}\right)$.

\section{THE CONCLUSION OF THE PROOF}

We have established at this point that we can compute the Weil homomorphism using a section $F: M \rightarrow P / O(n)$ which takes values in a measurable holonomy invariant subbundle with fiber $H / K$ (recall that $F: M \rightarrow P^{\prime} / K$ ). We now show that the Weil homomorphism factors through $H^{\cdot}(\mathfrak{h}, K)$.

Let $\tau \in \Lambda^{k}\left(\mathfrak{g l}(n)^{*}\right)_{O(n)}$; we can compute the Weil operator $\chi(\tau)$ by integrating forms in $\Omega_{c}^{m-k}(M, \mathscr{F})$ against the measurable tangential form $F^{*} \tau(\omega)=$ $\tau \circ \omega \circ F_{*}$. Notice that this last expression does not quite make sense since $F$ is a section of $P / O(n)$ and $\omega$ is a 1 -form on $P$. We can make sense of the expression, however, since $\tau$ is $O(n)$-basic, i.e., $\tau$ is defined on $\mathfrak{g l}(n) / \mathfrak{o}(n)$ and is $\operatorname{Ad} O(n)$ invariant. Thus if we consider $\omega \circ F_{*}$ as being defined "mod $O(n)$ ", we can evaluate $\tau$ on it. Now if $\tau \in \Lambda^{k}\left(\mathfrak{h}^{*}\right)_{K}$ we have the problem that $\omega \circ F_{*}$ may not take values in $\mathfrak{h} " \bmod O(n) "$, in which case we would not be able to evaluate $\tau$ on it. The next lemma shows that $\omega \circ F_{*}$ does take values in $\mathfrak{h} " \bmod O(n) "$.

Lemma 6.1. For any $\tau \in \Lambda^{k}(\mathfrak{h})_{K}, F^{*} \tau(\omega)$ is a well-defined tangential $k$-form on $M$.

Proof. Fix $x \in M$ and a coordinate chart $U_{\alpha}$ containing $x$. Then by a computation similar to one in the preceding section, we see that (in local coordinates on $P / O(n))$

$$
\left(\phi_{\alpha}\right)_{*} F(x)=f\left(x_{\alpha}\right) \sum_{\beta \in A} \lambda_{\beta}(x) \bar{\delta}\left(x_{\beta}, x_{\alpha}\right) .
$$

Let $t \mapsto x(t)$ be a $C^{1}$ path in the leaf through $x$ with $x(0)=x$. The functions $x \mapsto x_{\alpha}$ are locally constant along the leaves and $\bar{\delta}$ takes values in $H / K$ so

$$
\left(\phi_{\alpha}\right)_{*} F(x(t))=f\left(x_{\alpha}\right) \sum_{\beta \in A} \lambda_{\beta}(x(t)) \bar{\delta}\left(x_{\beta}, x_{\alpha}\right)
$$

is a path in $H / K$. It follows that for $X \in T_{x} \mathscr{F},\left(\phi_{\alpha}\right)_{*} F_{*} X \in T(H / K)$.

Now recall that the Bott connection $\omega$ is the weighted sum of the canonical connections on the local coordinate charts. Thus $\omega_{\alpha}: T P \mid U_{\alpha} \cong T U_{\alpha} \times$ $T G L(n, \mathbf{R}) \cong T U_{\alpha} \times G L(n, \mathbf{R}) \times \mathfrak{g l}(n) \rightarrow \mathfrak{g l}(n)$ is just projection on the last 
coordinate. Since $\left(\phi_{\alpha}\right)_{*} F_{*} X \in T(H / K)$ it follows that $\tau\left(\omega_{\alpha}\right)\left(\left(\phi_{\alpha}\right)_{*} F_{*} X\right)$ is defined. Since this is true for each $\alpha$ with $x \in U_{\alpha}$, it follows that $\tau(\omega)\left(F_{*} X\right)$ is defined.

It follows that for each $\tau \in \Lambda^{k}\left(\mathfrak{h}^{*}\right)_{K}$ the operator $\chi(\tau): \Omega_{c}^{m-k}(M \mathscr{F}) \rightarrow \mathbf{R}$ by

$$
\chi(\tau)(\omega)=\int_{M} F^{*} \tau(\omega) \wedge \omega
$$

is well defined. Moreover, if $\tau$ is the restriction to $\Lambda^{k}(\mathfrak{h})_{K}$ of an element $\tilde{\tau} \in \Lambda\left(\mathfrak{g l}(n)^{*}\right)_{O(n)}$, then $\chi(\tau)=\chi(\widetilde{\tau})$. It remains only to show that the map $\chi: \bigwedge^{k}\left(\mathfrak{h}^{*}\right)_{K} \rightarrow \Omega_{c}^{m-k}(M, \mathscr{F})^{\prime}$ is a map of differential algebras.

$$
\begin{aligned}
\chi\left(d_{\wedge} \tau\right)(\phi) & =\int_{M} F^{*} d_{\wedge} \tau(\omega) \wedge \phi=\int_{M}\left(d_{\mathscr{F}} F^{*} \tau(\omega)\right) \wedge \phi \\
& =\int_{M} \bar{d}\left(F^{*} \tau(\omega) \wedge \phi\right) \pm \int_{M} F^{*} \tau(\omega) \wedge d \phi \\
& = \pm \int_{M} F^{*} \tau(\omega) \wedge d \phi=\Lambda(\tau)(d \phi) .
\end{aligned}
$$

Thus $\chi$ factors through $H^{\cdot}(\mathfrak{h}, K)$ and this completes the proof of the Main Theorem.

\section{The Main COROllary}

To derive the Main Corollary from the Main Theorem we need the following key fact:

Theorem 7.1 [Z, S]. Let $G$ be a semisimple Lie group with finite center each of whose simple factors has real rank at least 2. Let $\Gamma \subset G$ be a lattice and suppose $\Gamma$ acts on a smooth manifold $M^{n}$ preserving a finite measure. Suppose, moreover, that either

(1) $M$ is compact and $\Gamma$ is cocompact, or

(2) $\Gamma$ is irreducible, the action of $\Gamma$ on $M$ is mixing, and the dimension of $M$ is smaller than the minimum dimension of an almost faithful real representation of $G$.

Then the derivative cocycle $\nu: \Gamma \times M \rightarrow G L(n, \mathbf{R})$ is measurably equivalent to a cocycle satisfying:

(i) $\delta$ takes values in a reductive algebraic subgroup $L=H \cdot K \subset G L(n, \mathbf{R})$ where $K$ is compact, $H$ is locally isomorphic to a semisimple factor of $G$, and both $K$ and $H$ are normal subgroups of $L . L$ is minimal in $\{H \subset G L(n, \mathbf{R}) \mid H$ is an algebraic subgroup and $\nu$ is equivalent to a cocycle into $H\}$.

(ii) The projection $\delta: \Gamma \times M \rightarrow L \rightarrow L / K$ is given (up to a finite covering) by a homomorphism of $\Gamma$ into $L / K$.

Set $N=K \backslash G \times \times_{\Gamma} M$ and let $\mathscr{F}$ be the natural foliation of $N$ transverse to the fibers of the bundle $p: N \rightarrow K \backslash G / \Gamma$. We will use the above theorem to show that $(N, \mathscr{F})$ satisfies the hypotheses of the Main Theorem. 
Let $\left\{V_{\alpha}, \psi_{\alpha}\right\}_{\alpha \in A_{1}}$ be a simple locally finite covering of $K \backslash G / \Gamma$ by regular coordinate charts and likewise $\left\{W_{\beta}, \eta_{\beta}\right\}_{\beta \in A_{2}}$ a covering of $M$. For each $\alpha \in A_{1}$ fix a point $x_{\alpha} \in V_{\alpha}$ and an identification $M \cong p^{-1}\left(x_{\alpha}\right)$. For each $z \in p^{-1}\left(x_{\alpha}\right)$, the connected component of $z$ in $L_{z} \cap p^{-1}\left(V_{\alpha}\right)$ is mapped diffeomorphically by $p$ onto $V_{\alpha}$. In this way we obtain a trivialization $p^{-1}\left(V_{\alpha}\right) \cong V_{\alpha} \times M$. Set $A=A_{1} \times A_{2}$ and for each $\alpha=\left(\alpha_{1}, \alpha_{2}\right) \in A$ set $U_{\alpha}=V_{\alpha_{1}} \times W_{\alpha_{2}}$, using the above trivialization to realize this as an open subset of $N$. Let $\phi_{\alpha}: U_{\alpha} \rightarrow \mathbf{R}^{m}=\mathbf{R}^{k} \times \mathbf{R}^{n}$ be the product map $\psi_{\alpha_{1}} \times \eta_{\alpha_{2}}$, where $k=\operatorname{dim}(G / K)$. Then $\left\{U_{\alpha}, \phi_{\alpha}\right\}$ is a simple locally finite covering of $M$ by regular foliation charts. Referring to the notation established in $\S 2$, we see that the generators $\left\{\gamma_{\alpha \beta}\right\}$ of the holonomy pseudogroup are given by the restrictions (in local coordinates) of elements of $\Gamma$ acting on $M$. In particular, the equivalence relation induced on $M$ by the orbits of $\Gamma$ is stably orbit equivalent (see, e.g., [Z2]) to the equivalence relation on the transversal $T$ induced by $\mathscr{F}$. Moreover, the linear holonomy cocycle on $T$ is related via a stable orbit equivalence to the derivative cocycle of the action of $\Gamma$ on $M$. Since the algebraic hull of a cocycle is an invariant of stable orbit equivalence [R], it follows from 7.1 that:

Corollary 7.2. The holonomy cocycle $\nu$ of $(N, \mathscr{F})$ is equivalent to a locally tempered cocycle $\delta$ into a reductive group $L=H \cdot K \subset G L(n, \mathbf{R})$, where $K$ is compact and $H$ is locally isomorphic to a semisimple factor of $G$.

Proof. We have explained everything save the local tempering. The local tempering follows from the fact that $K$ is compact and the projection of the cocycle of Theorem 7.1 into $L / K$ is given by a homomorphism of $\Gamma$ into $L / K$.

It remains now only to show that some conjugate of $L$ is stable under transpose.

Proposition 7.3. Let $L=H \cdot K \subset G L(n, \mathbf{R})$ where $H$ is semisimple, $K$ is compact and central in $L$, and $H$ and $K$ are connected. Then there is a Cartan involution of $G L(n, \mathbf{R})$ stabilizing $L$.

Proof. Note first that $L \subset S L(n, \mathbf{R})$. There is a decomposition $\mathbf{R}^{n}=V_{0} \oplus$ $V_{1} \oplus \cdots \oplus V_{k}$, where $H$ acts trivially on $V_{0}$ and irreducibly on each of the $V_{i}$ for $1 \leq i \leq k$. Let $G=G L\left(V_{0}\right) \times \mathbf{R}^{k}$. Then $G$ is the connected component of the identity of the centralizer of $H$ in $G L(n, \mathbf{R})$, where we consider each copy of $\mathbf{R}$ as acting by scalars on one of the $V_{i}, 1 \leq i \leq k$. Since $K$ is compact, connected, and centralizes $H$ it follows that $K \subset G L\left(V_{0}\right)$. Let $Q$ be a semisimple subgroup of $G L\left(V_{0}\right)$ containing $K$. By [Mo], there is a Cartan involution $\theta$ of $S L(n, \mathbf{R})$ which stabilizes $H \times Q$, and pointwise fixes $K$. Thus $\theta$ stabilizes $L$.

Now apply the Main Theorem to the foliation $(N, \mathscr{F})$ to deduce the Main Corollary. 


\section{REFERENCES}

[B1] R. Bott, On the characteristic classes of groups of diffeomorphisms, Enseign. Math. 23 (1977), 208-220.

[B2] - On a topological obstruction to integrability, Proc. Sympos. Pure Math., vol. 16, Amer. Math. Soc., Providence, R.I., 1970, pp. 27-36.

[B-H] R. Bott and A. Haefliger, On characteristic classes of $\Gamma$-foliations, Bull. Amer. Math. Soc. 78 (1972), 1039-1044.

[D] G. Duminy, L'invariant de Godbillon-Vey d'un feuilletage se localise dans les feuilles ressort, preprint (1982).

[F-M] J. Feldman and C. C. Moore, Ergodic equivalence relations, cohomology, and von Neumann algebras. I, Trans. Amer. Math. Soc. 234 (1977), 289-323.

[Ha] A. Haefliger, Sur les classes caractéristiques des feuilletages, Sém. Bourbaki, no. 412 (1971/ 72).

[H-H] J. Heitsch and S. Hurder, Secondary classes, Weil measures and the geometry of foliations, J. Differential Geom. 4 (1984).

[H-K] S. Hurder and A. Katok, Ergodic theory and Weil measures for foliations, Ann. of Math. (2) 126 (1987), 221-275.

[K-T] F. Kamber and P. Tondeur, Foliated bundles and characteristic classes, Lecture Notes in Math., Springer-Verlag, New York, 1976.

[K] H. Karcher, Riemannian center of mass and mollifier smoothing, Comm. Pure Appl. Math. 30 (1977), 509-541.

[M] G. A. Margulis, Discrete groups of motions of manifolds of non-positive curvature, Amer. Math. Soc. Transl. 109 (1977), 33-45.

[Mo] G. D. Mostow, Some new decomposition theorems for semi-simple groups, Mem. Amer. Math. Soc. 14 (1955), 31-54.

[R] A. Ramsay, Virtual groups and group actions, Adv. in Math. 6 (1971), 253-322.

[S] G. Stuck, Cocycles of ergodic group actions and vanishing of first cohomology for S-arithmetic groups, preprint.

[Z1] R. J. Zimmer, Ergodic theory and semisimple groups, Birkhäuser, Boston, Mass., 1984.

[Z2] _ Ergodic actions of semisimple groups and product relations, Ann. of Math. (2) 118 (1983), 9-19.

[Z3] _ On the algebraic hull of an automorphism group of a principal bundle, preprint (January, 1989).

Department of Mathematics, University of Maryland, College Park, Maryland 20742

Current address: Institut des Haute Etudes Scientiques, 35, route de Chartres, 91440 Buressur-Yvette 